\title{
Deep reflection-mode photoacoustic imaging and resolution scalability with depth
}

Kwang Hyun Song, Lihong V. Wang

Kwang Hyun Song, Lihong V. Wang, "Deep reflection-mode photoacoustic imaging and resolution scalability with depth," Proc. SPIE 6437, Photons Plus Ultrasound: Imaging and Sensing 2007: The Eighth Conference on Biomedical Thermoacoustics, Optoacoustics, and Acousto-optics, 643722 (13 February 2007); doi: 10.1117/12.698823

SPIE. Event: SPIE BiOS, 2007, San Jose, California, United States 


\title{
Deep reflection-mode photoacoustic imaging and resolution scalability with depth
}

\author{
Kwang Hyun Song and Lihong V. Wang \\ Optical Imaging Laboratory, Department of Biomedical Engineering, \\ Washington University in Saint Louis
}

\begin{abstract}
A deep reflection-mode photoacoustic (PA) imaging system was designed and implemented to visualize deep structures in biological tissues. To achieve good penetration depth, we chose near IR laser pulses at 804 nm wavelength for the generation of photoacoustic waves. To avoid overshadowing the deep PA signals by the surface PA signals, we employed dark-field illumination. To achieve good lateral resolution, we chose spherically focused high-numericalaperture ultrasonic transducers with $5 \mathrm{MHz}$ or $10 \mathrm{MHz}$ center frequencies. By using these transducers, we achieved 153 $\mu \mathrm{m}$ and $130 \mu \mathrm{m}$ axial resolutions, respectively, at $19.5 \mathrm{~mm}$ depth in $10 \%$ porcine gelatin containing $1 \%$ intralipid. The system was applied to imaging internal organs of small animals. Compared with our previous high-frequency (50-MHz) photoacoustic microscope, we scaled up the imaging depth while maintaining the ratio of the imaging depth to axial resolution more than 100. In addition, we studied the scalability of the imaging depth and the resolution with ultrasound frequency

Keywords: deep, reflection-mode, photoacoustic, brain cortex, kidney
\end{abstract}

\section{INTRODUCTION}

Photoacoustic imaging has been widely applied on biomedical imaging areas, such as angiography of blood vessels, angiogenesis studies, oxygenation monitoring of blood vessels, and early cancer detection since it is highly sensitive to intrinsic optical contrast, the blood vessels in the biological tissues. ${ }^{1-4}$ Photoacoustic imaging provides high spatial resolution and good contrast since it combines the benefit of ultrasonic and optical imaging modalities. In addition, it provides the great imaging depth in comparison with the pure optical imaging modalities because it is irrelevant to strong scattering of light in biological tissues.

On the basis of this imaging technology, photoacoustic tomography (PAT) has been developed and utilized on the brain imaging and whole head imaging of a small animal, and etc. ${ }^{5,6}$ Even though PAT has achieved high spatial resolution and low imaging artifacts, ${ }^{1,4 \sim 6}$ it is mostly suitable for imaging elevated objects, such as brain or breast, and it provides poor depth information because it integrates all the depth information that a transducer covers into a imaging plane. On the other hand, the reflection-mode (backward-mode) imaging is not limited by the shape of objects, ${ }^{7,8}$ but it may suffer from the strong photoacoustic signal generation from the object surface with a bright field illumination. Based on a reflection-mode method, Maslov et al developed a dark-field reflection-mode photoacoustic microscopy (PAM), ${ }^{9}$ which has achieved the axial resolution of up to $15 \mu \mathrm{m}$ and the lateral resolution of up to $45 \mu \mathrm{m}$ at $4 \mathrm{~mm}$ depth in 1\% agar gelatin containing $2 \%$ intralipid. In his study, the possible imaging depth has been claimed to be $3 \mathrm{~mm}$ and both resolutions will be degraded as the imaging depth increases. To go beyond this $3 \mathrm{~mm}$ limitation, we scaled up the PAM to a deep reflection-mode photoacoustic imaging system by employing its dark-field and donut shape illumination. At the same time, we kept the pixel count defined as the ratio of the imaging depth and axial resolution more than 100 to be comparable with the PAM which has the 200 pixel count. In addition, we experimentally show that the spatial resolution is scalable with its imaging depth.

In this study, we show the photoacoustic image of the brain cortex and the kidney of a rat. This technique will be able to provide functional information as well as structures of internal organs which are invisible with current optical imaging modalities.

Photons Plus Ultrasound: Imaging and Sensing 2007: The Eighth Conference on Biomedical Thermoacoustics, Optoacoustics, and Acousto-optics, edited by Alexander A. Oraevsky, Lihong V. Wang, Proc. of SPIE Vol. 6437, 643722, (2007) · 1605-7422/07/\$18 - doi: 10.1117/12.698823 


\section{SYSTEM DESIGN AND METHOD}

To achieve more than $3 \mathrm{~mm}$ imaging depth, described in the figure 1, we designed and built the deep reflection-mode photoacoustic imaging system. Figure 1 shows the schematic diagram of the system. The system mainly consists of two parts, optical part and mechanical part. To form the dark-field and donut-shape illumination, which reduce the overshadowing by the strong signal from the surface and maximize the detection of the weak signal from the deep regions, we used the concave lens, the spherical conical lens, and the optical condenser. To deliver high optical energy to the object, we employed two prisms instead of an optical fiber light delivery.

For light source, we used a Q-switch Nd:YAG laser (LS-2137/2, LOTIS TII) pumped tunable Ti:Sa laser (LT-2211A, LOTIS TII) as the excitation source which provides less than 15 -ns laser pulses with a $10 \mathrm{~Hz}$ pulse repetition rate. To achieve the deep penetration depth of light, we chose an $804 \mathrm{~nm}$ wavelength in the NIR range. This wavelength is also the isosbestic point for both oxy- and deoxy-hemoglobin absorption. We spread out the laser light sufficiently to meet the maximum permissible exposure (MPE) limit for skin at the $804 \mathrm{~nm}$ wavelength. At this wavelength, MPE is $31 \mathrm{~mJ} / \mathrm{cm}^{2} .10$

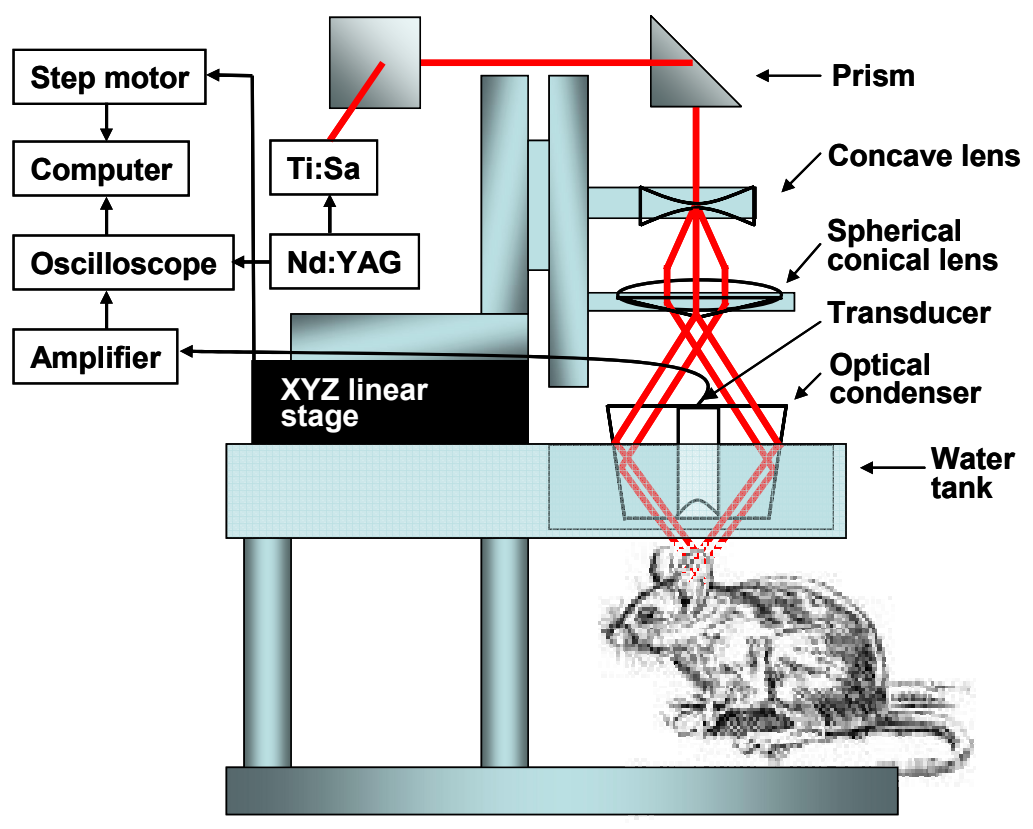

Figure.1: The schematic of the deep reflection-mode photoacoustic imaging system employing the dark-field illumination. The optical part is for the dark-field and donut-shape illumination and the mechanical part is for the raster scanning. The signals received by an ultrasonic transducer are digitized by an oscilloscope and collected by a computer.

To receive the weak photoacoustic signals from the locations deep within the animal and to achieve the deep imaging depth, we chose a $5 \mathrm{MHz}$ and a $10 \mathrm{MH}$ ultrasonic transducers, which have 1" focal length and 0.75 " active element (V308 and V315, Panametrics-NDT, Waltham, MA). They have a 72\% and a $64 \%$ nominal bandwidth, respectively. Both highly focused transducers are also critical to improve the lateral resolution, which is estimated by f-number $\lambda$. We chose these frequencies because low-frequency ultrasound experiences less attenuation than higher one and a wide active-element transducer has higher sensitivity than a narrow one. The transducer is immersed into water tank which has a $5 \mathrm{~cm} \times 5 \mathrm{~cm}$ opening which is sealed by the thin clear membrane. An animal is scanned through this opening and acoustically coupled with transducer by using acoustic gel.

We used an amplifier (5072PR, Panametrics-NDT) to amplify the photoacoustic signals received by the transducer and an oscilloscope (Tektronix TDS 5054) to digitize them at a rate of $125 \mathrm{MHz}$ with 5 times average. Finally, we stored all digitized signals on the computer, which also control a XY linear stage for the raster scanning. 


\section{EXPERIMENTAL RESULTS}

\subsection{Phantoms}

In order to evaluate SNR, resolution and imaging depth of the system, we imaged an around $50 \mu \mathrm{m}$ human hair fibers and an around $150 \mu \mathrm{m}$ horse hair fiber, both of which are sufficiently thin comparing with the wavelengths of the transducers. For SNR and axial resolution vs imaging depth, we imaged human hair fibers by using $5 \mathrm{MHz}$ and $10 \mathrm{MHz}$ ultrasonic transducers. The hair fibers were embedded at $4 \mathrm{~mm}, 8 \mathrm{~mm}, 16.5 \mathrm{~mm}$, and $19.5 \mathrm{~mm}$ in $10 \%$ porcine gelatin containing $1 \%$ intralipid. The reduced scattering coefficient of the tissue mimic phantom is approximately $10 \mathrm{~cm}^{-1},{ }^{11}$ which is close to the one of biological tissues.
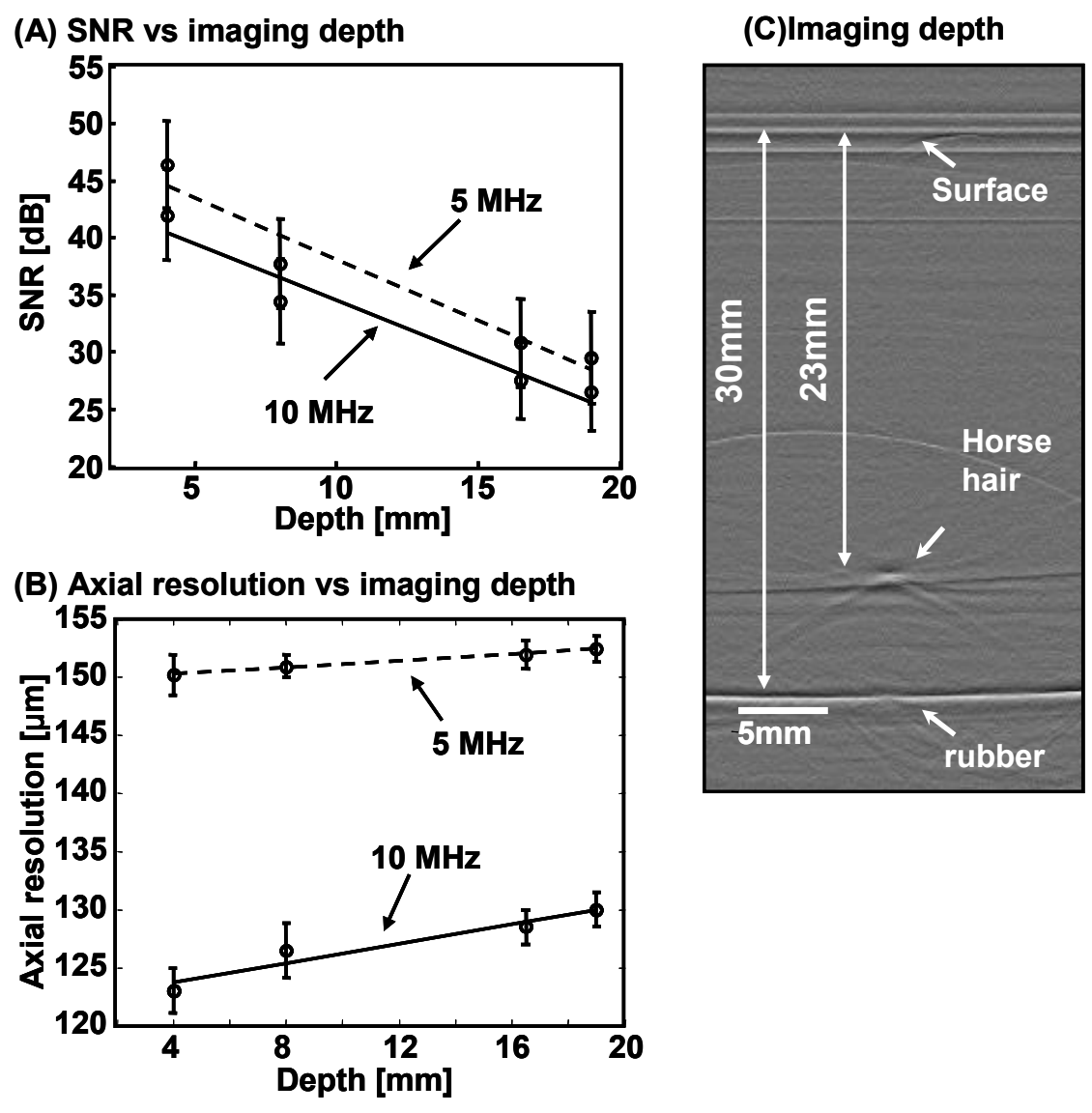

Figure 2. (A) and (B). SNR and resolution vs imaging depth. Obtained from around $50 \mu \mathrm{m}$ human hair fibers, which were embedded in $10 \%$ porcine gelatin containing $1 \%$ intralipid (The reduced scattering coefficient is approximately $10 \mathrm{~cm}^{-1}$ ). In the figure A, the maximum imaging depth will be approximately $25 \mathrm{~mm}$ with $10 \mathrm{MHz}$ transducer assuming minimum distinguishable SNR is $20 \mathrm{~dB}$, and with $5 \mathrm{MHz}$ transducer, it will be around $30 \mathrm{~mm}$. When calculating pixel count which is defined as imaging depth / resolution, $5 \mathrm{MHz}$ and $10 \mathrm{MHz}$ transducers give 127 and 150, respectively. Considering the photoacoustic microscope of 200 pixel count, the resolution is though to be scalable with the imaging depth. (C). Imaging depth in chicken breast tissue. Obtained from around $150 \mu \mathrm{m}$ horse hair fiber located around 23 $\mathrm{mm}$ deep. Measured SNR is around $24 \mathrm{~dB}$ at 30 times average. Even rubber plate located $30 \mathrm{~mm}$ deep was imaged.

Figure 2A shows the SNR vs imaging depth. With the $10 \mathrm{MHz}$ transducer, we achieved around $27 \mathrm{~dB}$ of SNR at $19.5 \mathrm{~mm}$. If the minimum SNR with which we can differentiate the object is $20 \mathrm{~dB}$, we will reach around $25 \mathrm{~mm}$ imaging depth based on the extrapolation. The $5 \mathrm{MHz}$ transducer provides higher SNR than the $10 \mathrm{MHz}$ one, which 
means that the imaging depth will go deeper to around $30 \mathrm{~mm}$. Figure B shows the axial resolution vs imaging depth. At $19.5 \mathrm{~mm}$ depth, we obtained $153 \mu \mathrm{m}$ and $130 \mu \mathrm{m}$ with $5 \mathrm{MHz}$ and $10 \mathrm{MHz}$ transducers, respectively. On the basis of these resolutions and imaging depths, we calculated pixel count which is defined as the ratio of the imaging depth and the resolution. The $5 \mathrm{MHz}$ and the $10 \mathrm{MHz}$ transducers provide 124 and 146, respectively. Considering that current dark-field photoacoustic microscope has 200 pixel counts, we claim that the resolution is scalable with the imaging depth.

To test the imaging depth more practically, we imaged an around $150 \mu \mathrm{m}$ horse hair located around $23 \mathrm{~mm}$ deep in chicken breast tissue from the tissue surface. The measured SNR from the horse hair is approximately $24 \mathrm{~dB}$ at 30 times average with in-house made $5 \mathrm{MHz}$ PVDF transducer. Even rubber plate located around $30 \mathrm{~mm}$ deep is clearly seen.

\subsection{In vivo noninvasive photoacoustic image of the brain cortex of a rat with skin and skull intact.}

For in vivo brain cortex imaging, Sprague Dolly rat (Harlen Co.) weighing around $200 \mathrm{~g}$ was employed. General anesthesia, which was a mixture of hydrochloride $(44 \mathrm{mg} / \mathrm{kg})$, xylazine hydrochloride $(2.5 \mathrm{mg} / \mathrm{kg})$, acepromazine maleate $(0.75 \mathrm{mg} / \mathrm{kg})$, and atropine $(0.025 \mathrm{mg} / \mathrm{kg})$, was subcutaneously administered to the rat every hour during the experiment. To minimize light scattering from the hair, the hair on the head was gently removed by using a commercial hair-removal lotion before imaging. During data acquisition, pure oxygen was provided through the mask.

Figure $3 \mathrm{~A}$ is the in vivo noninvasive maximum amplitude projection (MAP) photoacoustic image of the brain cortex of a rat with skin and skull intact. The thickness of the skin and the skull was totally $\sim 2 \mathrm{~mm}$. Figure $3 \mathrm{~B}$ is the corresponding invasive anatomical picture of the brain cortex. In the figure 3A, bright spots represent high optical absorption at the $804 \mathrm{~nm}$ wavelength. At this wavelength, the high optical absorbers are mainly blood vessels, which are marked with arrows and numbers. Number 1 to 6 indicate the blood vessels on the brain cortex of the left and right hemispheres, 7 shows the fissura transversa close to the posterior part, 8 denotes the blood vessels in the skull close to the anterior part, and 9 is the middle cerebral artery. These features are $\sim 3 \mathrm{~mm}$ below the skin surface and are well matched with the ones of the anatomical picture.

(A) Noninvasive MAP image of brain cortex

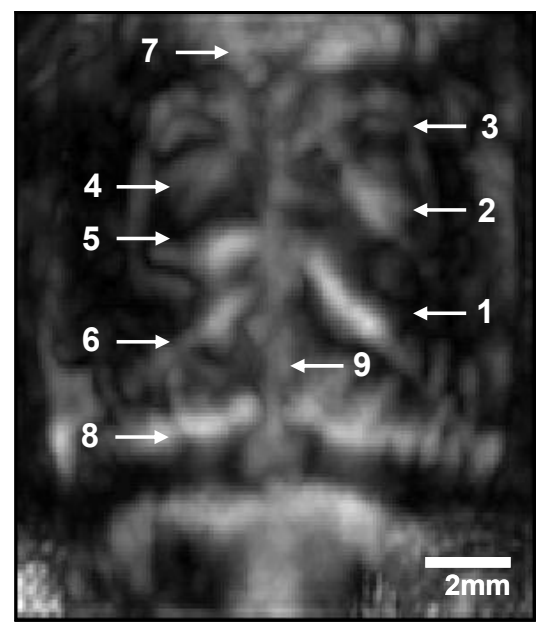

(B) Invasive brain cortex photo

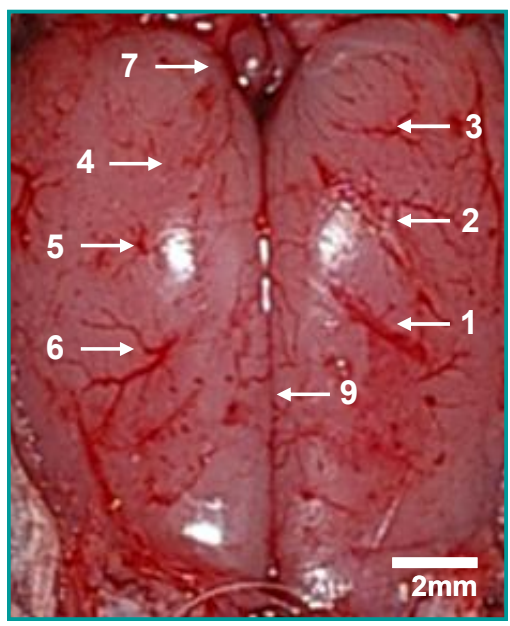

Figure 3. In vivo noninvasive photoacoustic image of the brain cortex of a rat with skin and skull intact. Figure $\mathrm{A}$ is the MAP image of the brain cortex and figure $\mathrm{B}$ is the corresponding anatomical picture obtained after photoacoustic imaging. The field of view is $12 \mathrm{~mm} \times 14 \mathrm{~mm}$. The predominant blood vessels are marked with arrows and numbers; 1 6. Blood vessels on the cortex surface; 7. Fissura transversa; 8 . Blood vessels in the skull; 9. Middle cerebral artery.

One of the strong advantages of the reflection-mode imaging is that it can provide the fine depth information comparing with photoacoustic tomography because its depth information can be obtained based on the time of arrival of 
ultrasound. Figure 4B shows the B-scan images of the brain cortex which are corresponding to the dotted lines in the figure 4A. On the B-scan images, the first layer indicates the skin, the second layer is the skull, the third layer represents the dura, and the fourth layer shows the brain cortex. The fissura transversa, the blood vessel on the brain cortex, the middle cerebral artery, and the blood vessel in the skull are distinguished and imaged clearly.

\section{(A) Noninvasive MAP image of brain cortex}

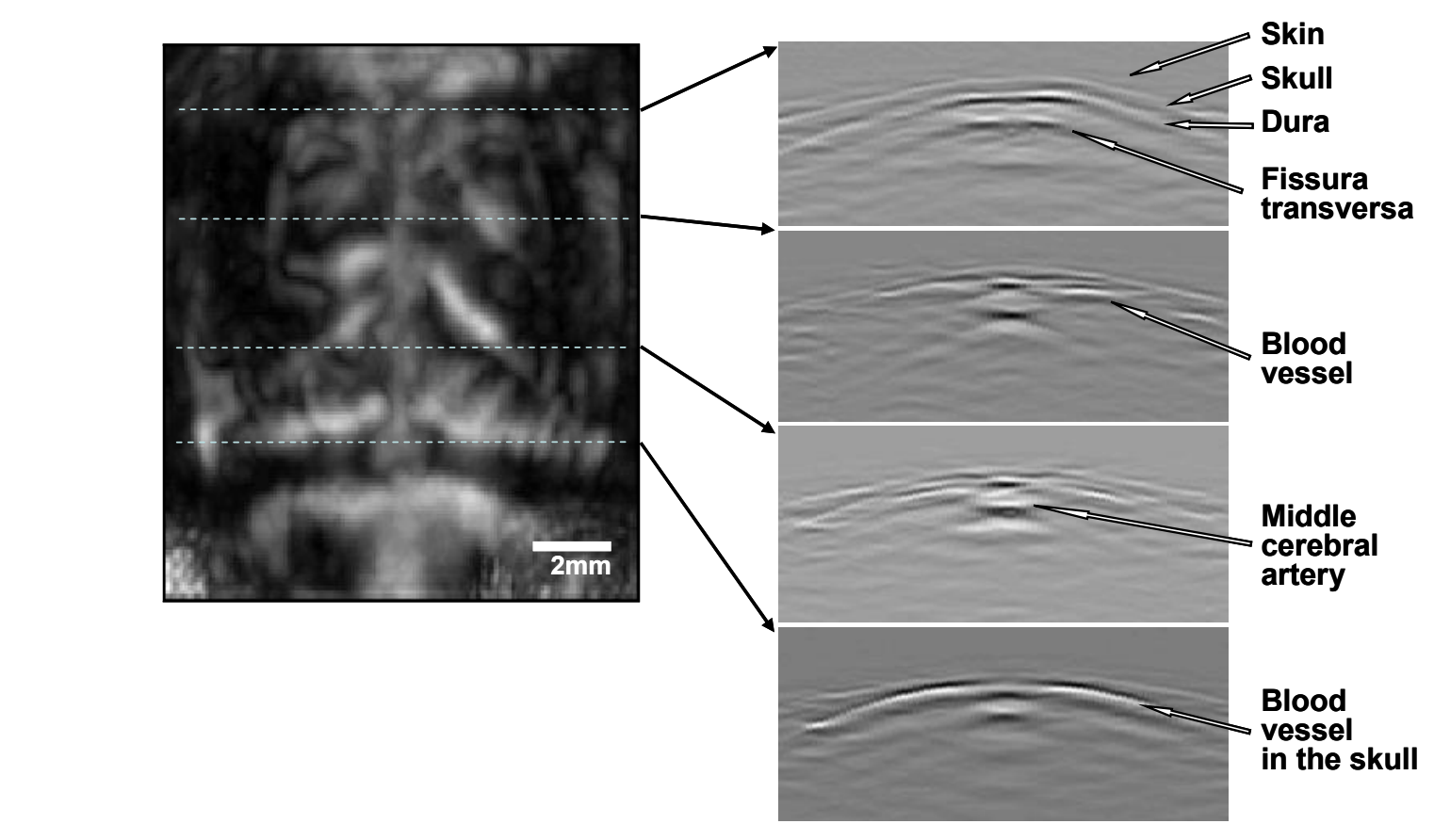

(B) B-scan images

Figure 4. B-scan images. B-scan images provide the depth information; the first layer is the skin; the second layer is the skull; the third layer is the dura; the fourth layer is the brain cortex.

\subsection{Noninvasive photoacoustic image of a rat kidney with skin intact.}

Another application of the deep reflection-mode photoacoustic imaging is internal organ imaging. Photoacoustic brain imaging, photoacoustic subcutaneous vasculature imaging, and etc. have been studied. However, the reflectionmode photoacoustic internal organ imaging has not been reported. Figure $5 \mathrm{~A}$ is the noninvasive MAP photoacoustic image of a rat kidney and figure 5B show the invasive anatomical picture of a rat kidney. Figure 5A shows the blood vessels in the skin and the kidney in deeper region. Figure 5C is the B-scan image corresponding to the dotted line in the figure 5A. This B-scan image shows how deep the blood vessels and the kidney locate. The blood vessels are located around $2 \sim 4 \mathrm{~mm}$ deep, and the kidney is located around $7 \mathrm{~mm}$ below the skin surface. Figure 5D is the three dimensional image of a rat kidney. It clearly displays the depth information of the blood vessels in the skin and the kidney at deeper depth.

\section{DISCUSSION}

Present imaging system has to be improved for various applications like time course measurement of the response to the internal or external stimuli. First, the total data acquisition time for a $20 \mathrm{~mm}$ x $14 \mathrm{~mm}$ three dimensional image is currently more than $2 \mathrm{hrs}$. Most of the time is for acquiring data by using the oscilloscope, not for changing the scanning position. To acquire data and control the motor, we used the LabVIEW (NI) which takes more time to 
initialize the oscilloscope right before every acquisition point. Therefore, we need to choose an acquisition mode like the fast frame mode, which require only one initialization. At this time, our goal is to speed up the acquisition time up to 30 minutes while keeping the same step size as current one. This time is mainly limited by the laser pulse repetition rate.

\section{(A) Noninvasive MAP image of rat kidney}

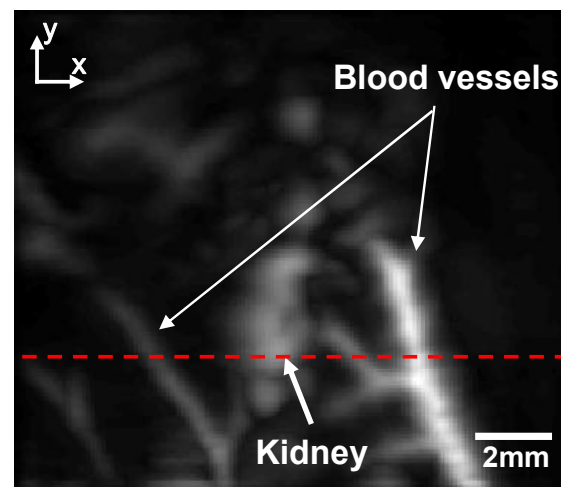

(C) B-scan image

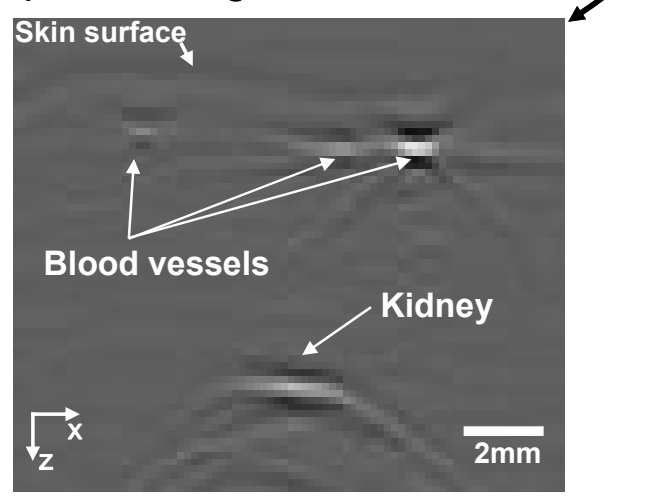

\section{(B) Invasive photo of rat kidney}

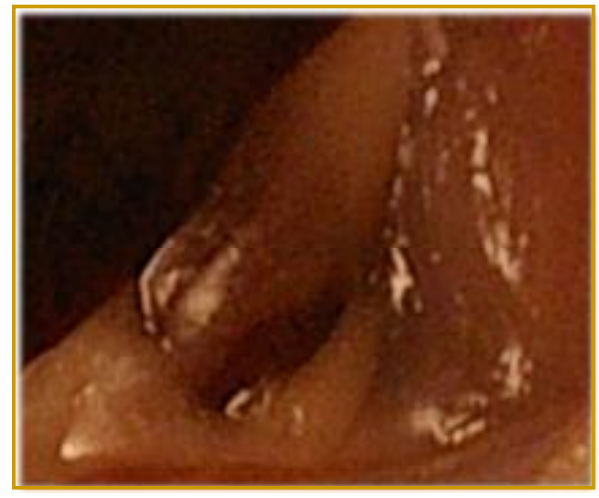

(D) Three dimensional image

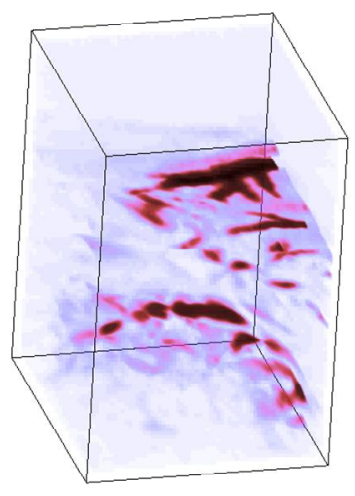

Figure 5. Noninvasive photoacoustic image of a rat kidney obtained skin and skull intact. Figure A: Noninvasive MAP image of a rat kidney; Figure B: Invasive anatomical picture of the rat kidney; Figure C: B-scan images of the rat kidney corresponding to the dotted line in the figure A; Figure D: Three dimensional image. The blood vessels are located around $2 \mathrm{~mm}$ below the skin surface. The kidney is located deeper around $7 \mathrm{~mm}$ from the skin surface.

Second, since this imaging system has a local illumination on the top surface of the object, the photoacoustic signal is mostly generated from the surface of the imaging target below the illumination spot. This effect happens more in highly absorptive object. Furthermore, in the deep internal organ imaging, light is easily blocked and absorbed by the adjacent organs, which causes less light arrival on the side of the organ. Due to these reasons, it is necessary to add more light sources from the side or other direction so that the targeted organ can absorb the optical energy from the side as well.

Third, internal organs unlike the brain are surrounded by other organs which may contain gas cavities. When ultrasound travels through these cavities, its speed will be changed resulting in an image distortion. In reflection-mode photoacoustic imaging, this image distortion will be less when no cavities exist between the object and the transducer. But, as expected, imaging deeper organs through these cavities will suffer from this distortion. In the long run, it is recommended to employ a method to correct this speed inhomogeneity for improving image quality.

Lastly, we can employ broader bandwidth ultrasonic transducers more than $10 \mathrm{MHz}$, which is currently used for brain cortex imaging, to improve spatial resolution. Obviously, we will lose the imaging depth, but in adequate imaging depth 
where the current 50-MHz photoacoustic microscope can't reach, this broader-bandwidth high-frequency deep imaging will be a good alternative to fill the gap between the $50 \mathrm{-MHz}$ photoacoustic microscope and the present deep reflectionmode photoacoustic imaging.

\section{CONCLUSION}

Deep reflection-mode photoacoustic imaging was successfully accomplished. The current $50-\mathrm{MHz}$ photoacoustic microscope provides a $3 \mathrm{~mm}$ imaging depth. This imaging depth may be sufficient for the clinical applications such as skin imaging. However, other applications like prostate cancer imaging, deep brain tumor imaging, and function brain imaging may need more than $3 \mathrm{~mm}$ imaging depth. To show feasibility of this deep imaging, we first imaged the brain cortex of a rat weighting around $200 \mathrm{~g}$. We imaged the rat kidney located around $7 \mathrm{~mm}$ below the skin surface. In addition, we showed experimentally that spatial resolution is scalable with imaging depth. Potentially, this imaging method will provide the functional information of internal organs.

\section{ACKNOWLEDGEMENT}

We thank Konstantine Maslov, Xinmai Yang for fruitful laboratory assistance. The project sponsored by National Institutes of Health grants R01 CA106728 and R01 NS46214 (BRP). L. Wang's email is lhwang@,biomed.wustl.edu.

\section{REFERENCE}

1. X. Wang, G. Ku, M. A. Wegiel, D. J. Bornhop, G. Stoica, and L.-H. V. Wang, "Noninvasive photoacoustic angiography of animal brains in vivo with near-infrared light and an optical contrast agent," Opt. Lett., Vol. 29, No. 7, 730-732 (2004).

2. A. A. Oraevsky, V. A. Andreev, A. A. Karabutov, D. R. Fleming, Z. Gatalica, H. Singh, and R. O. Esenaliev, "Laser opto-acoustic imaging of the breast: detection of cancer angiogenesis," Proc. SPIE 3597, 352, (1999).

3. R O Esenaliev, I V Larina, K V Larin, D J Deyo, M Motamedi and D S Prough, "Optoacoustic technique for noninvasive monitoring of blood oxygenation: a feasibility study," Appl. Opt. Vol. 41, 4722-31 (2002)

4. R. O. Esenaliev, A. A. Karabutov, and A. A. Oraevsky, "Sensitivity of Laser Opto-Acoustic Imaging in Detection of Small Deeply Embedded Tumors," IEEE J. Sel. T. Quantum Electron., Vol. 5, No. 4, 981-988 (1999).

5. X. Wang, Y. Pang, G. Ku, X. Xie, G. Stoica, and L.-H. V. Wang, "Noninvasive laser-induced photoacoustic tomography for structural and functional in vivo imaging of the brain," Nat. Biotechnol. Vol. 21, No. 7, 803-806 (2003).

6. K. H. Song, G. Stoica, and L. V. Wang, "In vivo three-dimensional photoacoustic tomography of a whole mouse head," Optics Letters 31, 2453-2455 (2006).

7. C. G. A. Hoelen, F. F. M. de Mul, R. Pongers, and A.Dekker, "Three-dimensional photoacoustic imaging of blood vessels in tissue," Opt. Lett, Vol. 23, No. 8, 648-650 (1998).

8. R. G. M. Kolkman, E. Honderbrink, W. Steenbergen, and F. F. M. de Mul, "In vivo photoacoustic imaging of blood vessels using an extreme-narrow aperture sensor," IEEE J. Sel. T. Quantum Electron. 9, 343 (2003).

9. K. Maslov, G. Stoica, L.-H. V. Wang, "In vivo dark-field reflection-mode photoacoustic microscopy," Opt. Lett., Vol 30, No. 6, 625-627, (2005).

10. American National Standards Institute, American national Standard for the Safe Use of Lasers ANSI Z136.1-2000 (American National Standards Institute, New York, 2000)

11. S. T. Flock, S. L. Jacques, B. C. Wilson, W. M. Star, M. J. C. van Gemert, Optical Properties of Intralipid: A phantom medium for light propagation studies, Lasers in Surg. Med. Vol. 12, No. 5, 510-519 (1992). 